\title{
PREPARATION AND EVALUATION OF POLYMERIC NANOPARTICLES OF GLIBENCLAMIDE
}

\author{
Naha Anup*, Kiran Sai , Rai Ikya, Vora Bhavisha, Reddy D Apoorva, P Kartika \\ Department of Pharmaceutics, Manipal College of Pharmaceutical Sciences, Manipal University, Manipal- 576104, \\ Karnataka, India. \\ *Corresponding AuthorE-mail: anupnaha@gmail.com
}

\begin{abstract}
Simple, reliable and reproducible method was used for the preparation of polymeric nanoparticles of Glibenclamide. The formulation was prepared by solvent evaporation method using magnetic stirrer with overnight stirring and the same was then evaluated for its particle size, drug content and in vitro dissolution studies. The above mentioned method showed similar particle size and exhibited an improvement in the drug entrapment efficiency. The ultraviolet spectrophotometric method was used to analyze Glibenclamide at $300 \mathrm{~nm}$ in different buffers. The study demonstrated the successful preparation of sustained release polymeric nanoparticles of Glibenclamide.
\end{abstract}

Keywords: Glibenclamide, polymeric nanoparticles, solvent evaporation

\section{INTRODUCTION}

Solubility is an important criterion for drug efficacy, independent of route of administration. It also poses a major challenge for pharmaceutical industries, which are developing new pharmaceutical products, since $40 \%$ of the active substances being identified are either insoluble or poorly soluble in aqueous media ${ }^{1}$. A limiting factor for in vivo performance of poorly water soluble drugs, following oral administration, is their resistance to being wetted and being dissolved into the fluid in the gastrointestinal tract ${ }^{1}$. Increasing the dissolution rate of poorly water soluble drugs is thus important for optimizing bioavailability ${ }^{1}$. To overcome these problems, various formulation strategies are reported in the literature including the use of surfactants (e.g. tween 80, gelucire), cyclodextrins (e.g. beta-cyclodextrin, hydroxypropyl beta-cyclodextrin), solid dispersions, micronization, lipid based systems ${ }^{2,3,4,5}$. However, these approaches are successful in only selected cases.

Nanoparticles are defined as particulate dispersions or solid particles with a size in the range of $10-1000 \mathrm{~nm}^{6}$. Nanocapsules are systems in which the drug is confined to a cavity surrounded by a unique polymer membrane, while nanospheres are matrix systems in which the drug is physically and uniformly dispersed ${ }^{6}$. Glibenclamide is one of the most widely used anti hyperglycemic drug. However, glibenclamide's low bioavailability has been attributed to its poor dissolution properties ${ }^{7}$. Hence, in the study it is planned to formulate polymeric nanoparticles of Glibenclamide to improve its dissolution and absorption.

\section{METHODS}

Preparation of polymeric nanoparticles of glibenclamide:

Weighed amount of drug was dissolved in dichloromethane, and weighed amount of Eudragit RLPO polymer was dissolved in methanol (Table 1). Drug-polymer solution was prepared by mixing the solutions, followed by vortexing. The drug-polymer solution was homogenized at 10000-13000 rpm and sonicated. The solution was then evaporated using a rotary evaporator, until there were no traces of the organic solvent. The resulting solution was centrifuged and the solution was freezed at $-80^{\circ} \mathrm{C}$ and then lyophilized, after the addition of required quantity of mannitol. 
Table 1: Formulation of various batches of polymeric nanoparticles

\begin{tabular}{|l|l|l|l|l|}
\hline \multirow{2}{*}{ Ingredient } & \multicolumn{4}{c|}{ Drug polymer ratio } \\
\cline { 2 - 5 } & $1: 5$ & $1: 10$ & $1: 20$ & $1: 40$ \\
\hline Drug(mg) & 5 & 5 & 5 & 5 \\
\hline Polymer(mg) & 25 & 50 & 100 & 200 \\
\hline
\end{tabular}

Optimized batches of the ratios 1:5 and 1:40 were prepared using the same procedure stated above.

\section{Evaluation of polymeric nanoparticles:}

\section{Particle size}

The particle sizes of the different formulations were measured using Malvern Zeta Sizer (Nano ZS), before and after lyophilization.

\section{Drug content}

$5 \mathrm{mg}$ of the lyophilized sample of each of the optimized formulations was weighed, dissolved in $5 \mathrm{ml}$ of the methanol, and the absorbance was measured spectrophotometrically at $300 \mathrm{~nm}$. The percentage entrapment was calculated.

\section{In vitro release}

The dissolution study was carried out using Type 2 dissolution with $900 \mathrm{ml}$ of phosphate buffer $(\mathrm{pH}$ 6.8) for 6 hours, first at intervals of 30 minutes, and then at intervals of an hour. The dissolution medium was kept in a thermostatically controlled water bath, maintained at $37 \pm 0.5^{0} \mathrm{C}$. The pre-weighed formulation was filled in a capsule, and then introduced into the dissolution jar. The paddle was rotated at $75 \mathrm{rpm}$. At different time intervals the samples were withdrawn and analyzed spectrophotometrically at $300 \mathrm{~nm}$ for drug release.

\section{RESULTS AND DISCUSSION}

\section{Particle size}

The particle sizes of the formulations were measured before and after lyophilization, and the results showed that particles were within the desired size range (Table 2,3).

Table 2: Particle size of formulations

\begin{tabular}{|c|c|c|}
\hline \multirow{2}{*}{ Drug polymer ratio } & \multicolumn{2}{|c|}{ Particle size $(\mathrm{nm})$} \\
\cline { 2 - 3 } & Before lyophilization & After lyophilization \\
\hline $1: 5$ & 154 & 2929 \\
\hline $1: 10$ & 95.4 & 2793 \\
\hline $1: 20$ & 100.4 & 1267 \\
\hline $1: 40$ & 133 & 1503 \\
\hline
\end{tabular}

Table 3: Particle size for Optimized Formulations

\begin{tabular}{|l|l|l|}
\hline \multirow{2}{*}{ Drug polymer ratio } & \multicolumn{2}{|c|}{ Particle size $(\mathrm{nm})$} \\
\cline { 2 - 3 } & Before lyophilization & After lyophilization \\
\hline $1: 5$ & 741.6 & 626.2 \\
\hline $1: 40$ & 554.5 & 683.6 \\
\hline
\end{tabular}

\section{Drug content}

The drug content of the optimized formulations was calculated, after measuring the absorbance at wavelength $300 \mathrm{~nm}$. The drug content of the formulation had a percentage entrapment in the range of $50-60 \%$.

\section{In vitro drug release}

Drug release profile of 1:4 and 1:5 drug polymer ratios was calculated. Dissolution study was performed for 1:4 and 1:5 drug polymer ratio and percentage cumulative drug release was calculated (\%CDD). The drug polymer ratio showed an increase in drug release with time. Formulation exhibited a complete drug release at the end of 6hrs (Figure 1) 


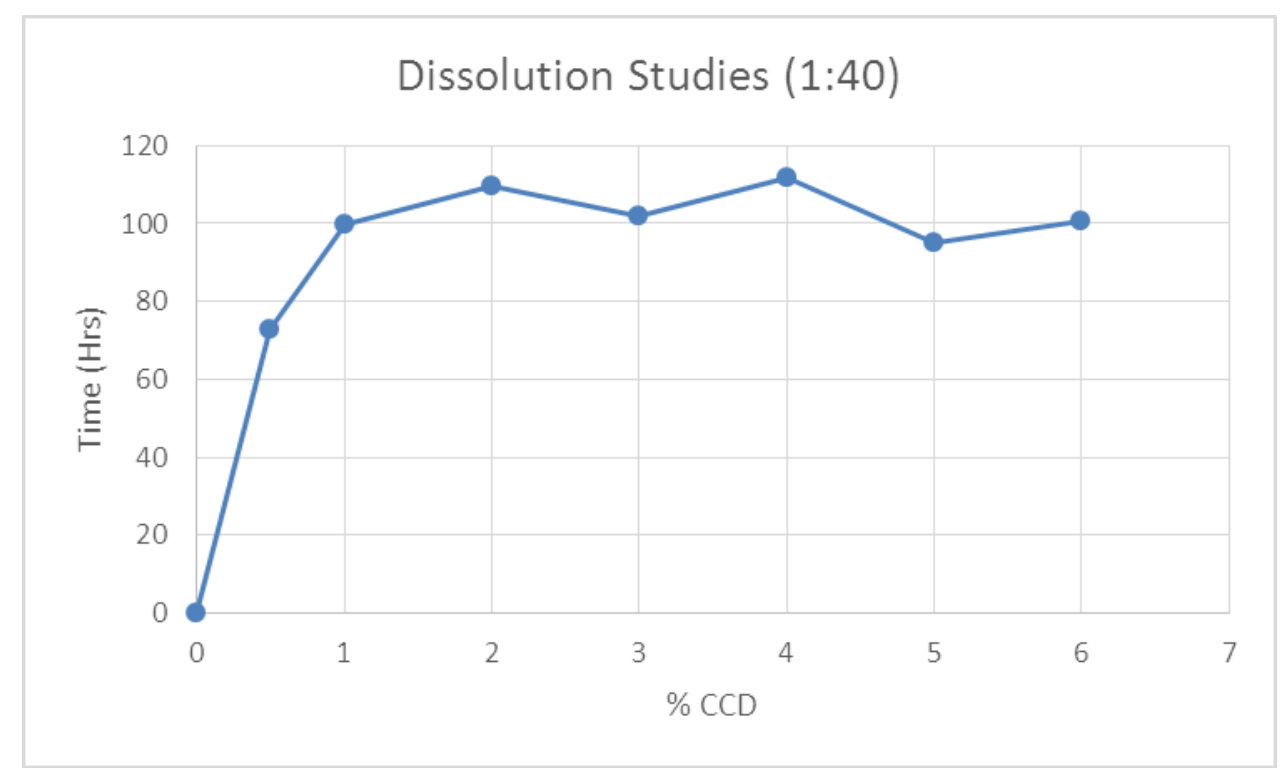

Figure 1: Drug release profile

\section{CONCLUSION}

The drug Glibenclamide was prepared by solvent evaporation method using magnetic stirrer with overnight stirring and the nanoparticles produced were evaluated. The optimized formulation prepared showed similar particle sizes and exhibited an improvement in

\section{REFERENCES}

1. Chander Parkash D, Singh SK, Kumar S, Datusalia AK, Development and characterization of Glibenclamide by solvent displacement method, Acta Poloniae Pharmaceutica and Drug Research, 2010, 67(3), 283-290.

2. Aungst BJ, Novel formulation strategies for improving oral bioavailability of drugs with poor membrane permeation or presystemic metabolism Pharm. Sci., 1993, 82, 979-987.

3. Burcham DL, Maurin MB, Hausner EA, Huang SM, Improved oral bioavailability of the hypocholesterolemic DMP 565 in dogs following oral dosing in oil and glycol solutions, Biopharm Drugs Dispos., 1997, 18,737-742.

4. Serajuddin AM, Shee PC, Mufson D, Bernstein DF, Augustine MA, Effect of vehicle amphiphilicity on the the drug entrapment efficiency. Formulation exhibited a complete drug release at the end of 6hrs. The present study demonstrated the successful preparation of sustained release polymeric nanoparticles of Glibenclamide. dissolution and bioavailability of a poorly water-soluble drug from solid dispersion. J Pharm Sci., 1988 77, 414-417.

5. Aungst BJ, Nguyen N, Rogers NJ, Rowe S, Hussain M, Shum L, White S, Improved oral bioavailability of an HIV protease inhibitor using Gelucire 44/14 and Labrasol vehicles, B T Gattefosse, 1994, 87, 49-54.

6. Mohanraj, Chen Y, Nanoparticles- A review, Tropical Journal of Pharmaceutical Research, 2006, 5(1), 561-573.

7. Behera A, Sahoo SK, Preparation and Evaluation of Glibenclamide - Loaded Biodegradable Nanoparticles, Trop J Pharm Res., 2013, 11 (3), 345. 